\title{
Some Common Coupled Fixed Point Results for Generalized Contraction in Complex-Valued Metric Spaces
}

\author{
Marwan Amin Kutbi, ${ }^{1}$ Akbar Azam, ${ }^{2}$ Jamshaid Ahmad, ${ }^{2}$ and Cristina Di Bari ${ }^{3}$ \\ ${ }^{1}$ Department of Mathematics, King Abdulaziz University, Jeddah, Saudi Arabia \\ ${ }^{2}$ Department of Mathematics, COMSATS Institute of Information Technology, Chak Shahzad, Islamabad, Pakistan \\ ${ }^{3}$ Università Degli Studi di Palermo, Dipartimento di Matematica e Informatica, Via Archirafi 34,90123 Palermo, Italy \\ Correspondence should be addressed to Jamshaid Ahmad; jamshaid_jasim@yahoo.com
}

Received 11 April 2013; Revised 22 May 2013; Accepted 22 May 2013

Academic Editor: Erdal Karapinar

Copyright (C) 2013 Marwan Amin Kutbi et al. This is an open access article distributed under the Creative Commons Attribution License, which permits unrestricted use, distribution, and reproduction in any medium, provided the original work is properly cited.

We introduce and study the notion of common coupled fixed points for a pair of mappings in complex valued metric space and demonstrate the existence and uniqueness of the common coupled fixed points in a complete complex-valued metric space in view of diverse contractive conditions. In addition, our investigations are well supported by nontrivial examples.

\section{Introduction}

Azam et al. [1] introduced the concept of complex-valued metric spaces and obtained sufficient conditions for the existence of common fixed points of a pair of contractive type mappings involving rational expressions. Subsequently, several authors have studied the existence and uniqueness of the fixed points and common fixed points of self-mappings in view of contrasting contractive conditions. Some of these investigations are noted in [2-26].

In [27], Bhaskar and Lakshmikantham introduced the concept of coupled fixed points for a given partially ordered set $X$. Recently Samet et al. $[28,29]$ proved that most of the coupled fixed point theorems (on ordered metric spaces) are in fact immediate consequences of well-known fixed point theorems in the literature. In this paper, we deal with the corresponding definition of coupled fixed point for mappings on a complex-valued metric space along with generalized contraction involving rational expressions. Our results extend and improve several fixed point theorems in the literature.

\section{Preliminaries}

Let $\mathbb{C}$ be the set of complex numbers and $z_{1}, z_{2} \in \mathbb{C}$. Define a partial order $\preceq$ on $\mathbb{C}$ as follows:

$$
z_{1} \preceq z_{2} \quad \text { iff } \operatorname{Re}\left(z_{1}\right) \leq \operatorname{Re}\left(z_{2}\right), \operatorname{Im}\left(z_{1}\right) \leq \operatorname{Im}\left(z_{2}\right) .
$$

Note that $0 \leq z_{1}, z_{2}$ and $z_{1} \neq z_{2}, z_{1} \leq z_{2}$ implies $\left|z_{1}\right|<\left|z_{2}\right|$.

Definition 1. Let $X$ be a nonempty set. Suppose that the selfmapping $d: X \times X \rightarrow \mathbb{C}$ satisfies the following:

(1) $0 \preceq d(x, y)$, for all $x, y \in X$ and $d(x, y)=0$ if and only if $x=y$;

(2) $d(x, y)=d(y, x)$ for all $x, y \in X$;

(3) $d(x, y) \preceq d(x, z)+d(z, y)$, for all $x, y, z \in X$. 
Then $d$ is called a complex valued metric on $X$, and $(X, d)$ is known as a complex valued metric space. A point $x \in X$ is called interior point of a set $A \subseteq X$ whenever, there exists $0 \prec r \in \mathbb{C}$ such that

$$
B(x, r)=\{y \in X: d(x, y) \prec r\} \subseteq A .
$$

A point $x \in X$ is a limit point of $A$ whenever, for every $0 \prec r \in \mathbb{C}$,

$$
B(x, r) \cap(A \backslash\{x\}) \neq \emptyset .
$$

$A$ is called open whenever each element of $A$ is an interior point of $A$. Moreover, a subset $B \subseteq X$ is called closed whenever each limit point of $B$ belongs to $B$. The family

$$
F=\{B(x, r): x \in X, 0 \prec r \in \mathbb{C}\}
$$

is a subbasis for a Hausdorff topology $\tau$ on $X$.

Let $\left\{x_{n}\right\}$ be a sequence in $X$ and $x \in X$. If for every $c \in \mathbb{C}$ with $0 \prec c$ there is $n_{0} \in \mathbb{N}$ such that, for all $n>n_{0}, d\left(x_{n}, x\right) \prec c$, then $\left\{x_{n}\right\}$ is said to be convergent, $\left\{x_{n}\right\}$ converges to $x$, and $x$ is the limit point of $\left\{x_{n}\right\}$. We denote this by $\lim _{n \rightarrow+\infty} x_{n}=x$, or $x_{n} \rightarrow x$, as $n \rightarrow+\infty$. If for every $c \in \mathbb{C}$ with $0 \prec c$ there is $n_{0} \in \mathbb{N}$ such that, for all $n>n_{0}, d\left(x_{n}, x_{n+m}\right) \prec c$, then $\left\{x_{n}\right\}$ is called a Cauchy sequence in $(X, d)$. If every Cauchy sequence is convergent in $(X, d)$, then $(X, d)$ is called a complete complex valued metric space. We require the following lemmas.

Lemma 2 (see [1]). Let $(X, d)$ be a complex valued metric space, and let $\left\{x_{n}\right\}$ be a sequence in $X$. Then $\left\{x_{n}\right\}$ converges to $x$ if and only if $\left|d\left(x_{n}, x\right)\right| \rightarrow 0$ as $n \rightarrow+\infty$.

Lemma 3 (see [1]). Let $(X, d)$ be a complex valued metric space, and let $\left\{x_{n}\right\}$ be a sequence in $X$. Then $\left\{x_{n}\right\}$ is a Cauchy sequence if and only if $\left|d\left(x_{n}, x_{n+m}\right)\right| \rightarrow 0$ as $n \rightarrow+\infty$.

Definition 4 (see [27]). An element $(x, y) \in X \times X$ is called a coupled fixed point of $T: X \times X \rightarrow X$ if

$$
x=T(x, y), \quad y=T(y, x) .
$$

Definition 5. An element $(x, y) \in X \times X$ is called a coupled coincidence point of $S, T: X \times X \rightarrow X$ if

$$
S(x, y)=T(x, y), \quad S(y, x)=T(y, x) .
$$

Example 6. Let $X=\mathbb{R}$ and $S, T: X \times X \rightarrow X$ defined as $S(x, y)=x^{2} y^{2}$ and $T(x, y)=(4 / 3)(x+y)$ for all $x, y \in X$. Then $(0,0),(1,2)$, and $(2,1)$ are coupled coincidence points of $S$ and $T$.

Example 7. Let $X=\mathbb{R}$ and $S, T: X \times X \rightarrow X$ defined as $S(x, y)=x+y+\sin (x+y)$ and $T(x, y)=x+y+x y+\cos (x+$ $y$ ) for all $x, y \in X$. Then $(0, \pi / 4)$ and $(\pi / 4,0)$ are coupled coincidence points of $S$ and $T$.

Definition 8. An element $(x, y) \in X \times X$ is called a common coupled fixed point of $S, T: X \times X \rightarrow X$ if

$$
x=S(x, y)=T(x, y), \quad y=S(y, x)=T(y, x) .
$$

Example 9. Let $X=\mathbb{R}$ and $S, T: X \times X \rightarrow X$ defined as $S(x, y)=x\left(\left(x+(y-1)^{2}\right) / 2\right)$ and $T(x, y)=x\left(\sqrt{x^{2}+y^{2}+4}-2\right)$ for all $x, y \in X$. Then $(0,0),(1,2)$, and $(2,1)$ are common coupled fixed points of $S$ and $T$.

In the following, we provide common coupled fixed point theorem for a pair of mappings satisfying a rational inequality in complex valued metric spaces.

Theorem 10. Let $(X, d)$ be a complete complex-valued metric space, and let the mappings $S, T: X \times X \rightarrow X$ satisfy

$$
\begin{aligned}
d(S(x, y), T(u, v)) \leq & \frac{\alpha(d(x, u)+d(y, v))}{2} \\
& +(\beta d(x, S(x, y)) d(u, T(u, v)) \\
& +\gamma d(u, S(x, y)) d(x, T(u, v))) \\
& \times(1+d(x, u)+d(y, v))^{-1}
\end{aligned}
$$

for all $x, y, u, v \in X$ and $\alpha, \beta$, and $\gamma$ are nonnegative reals with $\alpha+\beta+\gamma<1$. Then $S$ and $T$ have a unique common coupled fixed point.

Proof. Let $x_{0}$ and $y_{0}$ be arbitrary points in $X$. Define $x_{2 k+1}=$ $S\left(x_{2 k}, y_{2 k}\right), y_{2 k+1}=S\left(y_{2 k}, x_{2 k}\right)$ and $x_{2 k+2}=T\left(x_{2 k+1}, y_{2 k+1}\right)$, $y_{2 k+2}=T\left(y_{2 k+1}, x_{2 k+1}\right)$, for $k=0,1, \ldots$

Then,

$$
\begin{aligned}
d\left(x_{2 k+1}, x_{2 k+2}\right) & \\
= & d\left(S\left(x_{2 k}, y_{2 k}\right), T\left(x_{2 k+1}, y_{2 k+1}\right)\right) \\
\leq & \frac{\alpha\left(d\left(x_{2 k}, x_{2 k+1}\right)+d\left(y_{2 k}, y_{2 k+1}\right)\right)}{2} \\
+ & \left(\beta d\left(x_{2 k}, S\left(x_{2 k}, y_{2 k}\right)\right)\right. \\
& \left.\times d\left(x_{2 k+1}, T\left(x_{2 k+1}, y_{2 k+1}\right)\right)\right) \\
& \times\left(1+d\left(x_{2 k}, x_{2 k+1}\right)+d\left(y_{2 k}, y_{2 k+1}\right)\right)^{-1} \\
+ & \left(\gamma d\left(x_{2 k+1}, S\left(x_{2 k}, y_{2 k}\right)\right)\right. \\
& \times\left(1+d\left(x_{2 k}, T\left(x_{2 k+1}, y_{2 k+1}\right)\right)\right) \\
\leq & \frac{\alpha\left(d\left(x_{2 k}, x_{2 k+1}\right)+d\left(y_{2 k}, y_{2 k+1}\right)\right)}{2} \\
+ & \frac{\beta d\left(x_{2 k+1}, x_{2 k+1}\right) d\left(x_{2 k}, x_{2 k+2}\right)}{1+d\left(x_{2 k}, x_{2 k+1}\right)+d\left(y_{2 k}, y_{2 k+1}\right)} \\
& \left.\quad x_{2 k+1}\right) d\left(x_{2 k+1}, x_{2 k+2}\right) \\
& +d\left(x_{2 k}, x_{2 k+1}\right)+d\left(y_{2 k}, y_{2 k+1}\right)
\end{aligned}
$$




$$
\begin{aligned}
\leq & \frac{\alpha\left(d\left(x_{2 k}, x_{2 k+1}\right)+d\left(y_{2 k}, y_{2 k+1}\right)\right)}{2} \\
& +\frac{\beta d\left(x_{2 k}, x_{2 k+1}\right) d\left(x_{2 k+1}, x_{2 k+2}\right)}{1+d\left(x_{2 k}, x_{2 k+1}\right)+d\left(y_{2 k}, y_{2 k+1}\right)},
\end{aligned}
$$

which implies that

$$
\begin{aligned}
\left|d\left(x_{2 k+1}, x_{2 k+2}\right)\right| \leq & \frac{\alpha\left|d\left(x_{2 k}, x_{2 k+1}\right)+d\left(y_{2 k}, y_{2 k+1}\right)\right|}{2} \\
& +\frac{\beta\left|d\left(x_{2 k}, x_{2 k+1}\right) d\left(x_{2 k+1}, x_{2 k+2}\right)\right|}{\left|1+d\left(x_{2 k}, x_{2 k+1}\right)+d\left(y_{2 k}, y_{2 k+1}\right)\right|} .
\end{aligned}
$$

Since $\left|1+d\left(x_{2 k}, x_{2 k+1}\right)+d\left(y_{2 k}, y_{2 k+1}\right)\right|>\left|d\left(x_{2 k}, x_{2 k+1}\right)\right|$, so we get

$$
\begin{aligned}
& \left|d\left(x_{2 k+1}, x_{2 k+2}\right)\right| \\
& \leq \frac{\alpha\left|d\left(x_{2 k}, x_{2 k+1}\right)\right|+\alpha\left|d\left(y_{2 k}, y_{2 k+1}\right)\right|}{2} \\
& \quad+\beta\left|d\left(x_{2 k+1}, x_{2 k+2}\right)\right|,
\end{aligned}
$$

and hence

$$
\begin{aligned}
&\left|d\left(x_{2 k+1}, x_{2 k+2}\right)\right| \\
& \leq \frac{1}{2}\left(\frac{\alpha}{1-\beta}\right)\left|d\left(x_{2 k}, x_{2 k+1}\right)\right| \\
& \quad+\frac{1}{2}\left(\frac{\alpha}{1-\beta}\right)\left|d\left(y_{2 k}, y_{2 k+1}\right)\right| .
\end{aligned}
$$

Similarly, one can show that

$$
\begin{aligned}
\left|d\left(y_{2 k+1}, y_{2 k+2}\right)\right| \leq & \frac{1}{2}\left(\frac{\alpha}{1-\beta}\right)\left|d\left(y_{2 k}, y_{2 k+1}\right)\right| \\
& +\frac{1}{2}\left(\frac{\alpha}{1-\beta}\right)\left|d\left(x_{2 k}, x_{2 k+1}\right)\right| .
\end{aligned}
$$

Also,

$$
\begin{aligned}
& d\left(x_{2 k+2}, x_{2 k+3}\right) \\
& =d\left(T\left(x_{2 k+1}, y_{2 k+1}\right), S\left(x_{2 k+2}, y_{2 k+2}\right)\right) \\
& \leq \frac{\alpha\left(d\left(x_{2 k+1}, x_{2 k+2}\right)+d\left(y_{2 k+1}, y_{2 k+2}\right)\right)}{2} \\
& +\left(\beta d\left(x_{2 k+1}, T\left(x_{2 k+1}, y_{2 k+1}\right)\right)\right. \\
& \left.\times d\left(x_{2 k+2}, S\left(x_{2 k+2}, y_{2 k+2}\right)\right)\right) \\
& \times\left(1+d\left(x_{2 k+1}, x_{2 k+2}\right)+d\left(y_{2 k+1}, y_{2 k+2}\right)\right)^{-1} \\
& +\left(\gamma d\left(x_{2 k+2}, T\left(x_{2 k+1}, y_{2 k+1}\right)\right)\right. \\
& \left.\times d\left(x_{2 k+1}, S\left(x_{2 k+2}, y_{2 k+2}\right)\right)\right) \\
& \times\left(1+d\left(x_{2 k+1}, x_{2 k+2}\right)+d\left(y_{2 k+1}, y_{2 k+2}\right)\right)^{-1} \\
& \leq \frac{\alpha\left(d\left(x_{2 k+1}, x_{2 k+2}\right)+d\left(y_{2 k+1}, y_{2 k+2}\right)\right)}{2} \\
& +\frac{\beta d\left(x_{2 k+1}, x_{2 k+2}\right) d\left(x_{2 k+2}, x_{2 k+3}\right)}{1+d\left(x_{2 k+1}, x_{2 k+2}\right)+d\left(y_{2 k+1}, y_{2 k+2}\right)} \\
& +\frac{\gamma d\left(x_{2 k+2}, x_{2 k+2}\right) d\left(x_{2 k+1}, x_{2 k+3}\right)}{1+d\left(x_{2 k+1}, x_{2 k+2}\right)+d\left(y_{2 k+1}, y_{2 k+2}\right)} \\
& \preceq \frac{\alpha\left(d\left(x_{2 k+1}, x_{2 k+2}\right)+d\left(y_{2 k+1}, y_{2 k+2}\right)\right)}{2} \\
& +\frac{\beta d\left(x_{2 k+1}, x_{2 k+2}\right) d\left(x_{2 k+2}, x_{2 k+3}\right)}{1+d\left(x_{2 k+1}, x_{2 k+2}\right)+d\left(y_{2 k+1}, y_{2 k+2}\right)},
\end{aligned}
$$

so that

$$
\begin{aligned}
\left|d\left(x_{2 k+2}, x_{2 k+3}\right)\right| \leq & \frac{\alpha\left|d\left(x_{2 k+1}, x_{2 k+2}\right)+d\left(y_{2 k+1}, y_{2 k+2}\right)\right|}{2} \\
& +\frac{\beta\left|d\left(x_{2 k+1}, x_{2 k+2}\right)\right|\left|d\left(x_{2 k+2}, x_{2 k+3}\right)\right|}{\left|1+d\left(x_{2 k+1}, x_{2 k+2}\right)+d\left(y_{2 k+1}, y_{2 k+2}\right)\right|} .
\end{aligned}
$$

As $\left|1+d\left(x_{2 k+1}, x_{2 k+2}\right)+d\left(y_{2 k+1}, y_{2 k+2}\right)\right|>\left|d\left(x_{2 k+1}, x_{2 k+2}\right)\right|$, therefore

$$
\begin{aligned}
\left|d\left(x_{2 k+2}, x_{2 k+3}\right)\right| \leq & \frac{1}{2}\left(\frac{\alpha}{1-\beta}\right)\left|d\left(x_{2 k+1}, x_{2 k+2}\right)\right| \\
& +\frac{1}{2}\left(\frac{\alpha}{1-\beta}\right)\left|d\left(y_{2 k+1}, y_{2 k+2}\right)\right| .
\end{aligned}
$$

Similarly, one can show that

$$
\begin{aligned}
\left|d\left(y_{2 k+2}, y_{2 k+3}\right)\right| \leq & \frac{\alpha}{1-\beta}\left|d\left(y_{2 k+1}, y_{2 k+2}\right)\right| \\
& +\frac{1}{2}\left(\frac{\alpha}{1-\beta}\right)\left|d\left(x_{2 k+1}, x_{2 k+2}\right)\right| .
\end{aligned}
$$


Adding (12)-(17), we get

$$
\begin{aligned}
& \left|d\left(x_{2 k+1}, x_{2 k+2}\right)\right|+\left|d\left(y_{2 k+1}, y_{2 k+2}\right)\right| \\
& \quad \leq \frac{\alpha}{1-\beta}\left|d\left(x_{2 k}, x_{2 k+1}\right)\right|+\frac{\alpha}{1-\beta}\left|d\left(y_{2 k}, y_{2 k+1}\right)\right| \\
& \left|d\left(x_{2 k+2}, x_{2 k+3}\right)\right|+\left|d\left(y_{2 k+2}, y_{2 k+3}\right)\right| \\
& \quad \leq \frac{\alpha}{1-\beta}\left|d\left(x_{2 k+1}, x_{2 k+2}\right)\right|+\frac{\alpha}{1-\beta}\left|d\left(y_{2 k+1}, y_{2 k+2}\right)\right| .
\end{aligned}
$$

If $h=\alpha /(1-\beta)<1$, then from (18), we get

$$
\begin{aligned}
& \left|d\left(x_{n}, x_{n+1}\right)\right|+\left|d\left(y_{n}, y_{n+1}\right)\right| \\
& \leq h\left(\left|d\left(x_{n-1}, x_{n}\right)\right|+\left|d\left(y_{n-1}, y_{n}\right)\right|\right) \\
& \leq \cdots \leq h^{n}\left(\left|d\left(x_{0}, x_{1}\right)\right|+\left|d\left(y_{0}, y_{1}\right)\right|\right) .
\end{aligned}
$$

Now if $\left|d\left(x_{n}, x_{n+1}\right)\right|+\left|d\left(y_{n}, y_{n+1}\right)\right|=\delta_{n}$, then

$$
\delta_{n} \leq h \delta_{n-1} \leq \cdots \leq h^{n} \delta_{0}
$$

Without loss of generality, we take $m>n$. Since $0 \leq h<1$, so we get

$$
\begin{aligned}
& \left|d\left(x_{n}, x_{m}\right)\right|+\left|d\left(y_{n}, y_{m}\right)\right| \\
& \leq\left|d\left(x_{n}, x_{n+1}\right)\right|+\left|d\left(y_{n}, y_{n+1}\right)\right|+\cdots \\
& \quad+\left|d\left(x_{m-1}, x_{m}\right)\right|+\left|d\left(y_{m-1}, y_{m}\right)\right| \\
& \leq\left[h^{n} \delta_{0}+h^{n+1} \delta_{0}+\cdots+h^{m-1} \delta_{0}\right] \\
& \leq \sum_{i=n}^{m-1} h^{i} \delta_{0} \longrightarrow 0, \quad \text { as } m, n \longrightarrow+\infty .
\end{aligned}
$$

This implies that $\left\{x_{n}\right\}$ and $\left\{y_{n}\right\}$ are Cauchy sequences in $X$. Since $X$ is complete, there exists $x, y \in X$ such that $x_{n} \rightarrow x$ and $y_{n} \rightarrow y$ as $n \rightarrow+\infty$. We now show that $x=S(x, y)$ and $y=S(y, x)$. We suppose on the contrary that $x \neq S(x, y)$ and $y \neq S(y, x)$ so that $0 \prec d(x, S(x, y))=l_{1}$ and $0 \prec$ $d(y, S(y, x))=l_{2}$; we would then have

$$
\begin{aligned}
l_{1}= & d(x, S(x, y)) \preceq d\left(x, x_{2 k+2}\right)+d\left(x_{2 k+2}, S(x, y)\right) \\
\preceq & d\left(x, x_{2 k+2}\right)+d\left(T\left(x_{2 k+1}, y_{2 k+1}\right), S(x, y)\right) \\
\preceq & d\left(x, x_{2 k+2}\right)+\frac{\alpha\left(d\left(x_{2 k+1}, x\right)+d\left(y_{2 k+1}, y\right)\right)}{2} \\
& +\frac{\beta d\left(x_{2 k+1}, T\left(x_{2 k+1}, y_{2 k+1}\right)\right) d(x, S(x, y))}{1+d\left(x_{2 k+1}, x\right)+d\left(y_{2 k+1}, y\right)} \\
& +\frac{\gamma d\left(x, T\left(x_{2 k+1}, y_{2 k+1}\right)\right) d\left(x_{2 k+1}, S(x, y)\right)}{1+d\left(x_{2 k+1}, x\right)+d\left(y_{2 k+1}, y\right)} \\
= & d\left(x, x_{2 k+2}\right)+\frac{\alpha\left(d\left(x_{2 k+1}, x\right)+d\left(y_{2 k+1}, y\right)\right)}{2} \\
& +\frac{\beta d\left(x_{2 k+1}, x_{2 k+2}\right) d(x, S(x, y))}{1+d\left(x_{2 k+1}, x\right)+d\left(y_{2 k+1}, y\right)} \\
& +\frac{\gamma d\left(x, x_{2 k+2}\right) d\left(x_{2 k+1}, S(x, y)\right)}{1+d\left(x_{2 k+1}, x\right)+d\left(y_{2 k+1}, y\right)}
\end{aligned}
$$

so that

$$
\begin{aligned}
\left|l_{1}\right| \leq & \left|d\left(x, x_{2 k+2}\right)\right|+\frac{\alpha\left|d\left(x_{2 k+1}, x\right)+d\left(y_{2 k+1}, y\right)\right|}{2} \\
& +\frac{\beta\left|d\left(x_{2 k+1}, x_{2 k+2}\right)\right||d(x, S(x, y))|}{\left|1+d\left(x_{2 k+1}, x\right)+d\left(y_{2 k+1}, y\right)\right|} \\
& +\frac{\gamma\left|d\left(x, x_{2 k+2}\right)\right|\left|d\left(x_{2 k+1}, S(x, y)\right)\right|}{\left|1+d\left(x_{2 k+1}, x\right)+d\left(y_{2 k+1}, y\right)\right|}
\end{aligned}
$$

By taking $k \rightarrow+\infty$, we get $|d(x, S(x, y))|=0$ which is a contradiction so that $x=S(x, y)$. Similarly, one can prove that $y=S(y, x)$. It follows similarly that $x=T(x, y)$ and $y=T(y, x)$. So we have proved that $(x, y)$ is a common coupled fixed point of $S$ and $T$. We now show that $S$ and $T$ have a unique common coupled fixed point. For this, assume that $\left(x^{*}, y^{*}\right) \in X$ is a second common coupled fixed point of $S$ and $T$. Then

$$
\begin{aligned}
d\left(x, x^{*}\right)= & d\left(S(x, y), T\left(x^{*}, y^{*}\right)\right) \\
\preceq & \frac{\alpha\left(d\left(x, x^{*}\right)+d\left(y, y^{*}\right)\right)}{2} \\
& +\frac{\beta d(x, S(x, y)) d\left(x^{*}, T\left(x^{*}, y^{*}\right)\right)}{1+d\left(x, x^{*}\right)+d\left(y, y^{*}\right)} \\
& +\frac{\gamma d\left(x, T\left(x^{*}, y^{*}\right)\right) d\left(x^{*}, S(x, y)\right)}{1+d\left(x, x^{*}\right)+d\left(y, y^{*}\right)}
\end{aligned}
$$




$$
\begin{aligned}
\preceq & \frac{\alpha\left(d\left(x, x^{*}\right)+d\left(y, y^{*}\right)\right)}{2} \\
& +\frac{\beta d(x, x) d\left(x^{*}, x^{*}\right)}{1+d\left(x, x^{*}\right)+d\left(y, y^{*}\right)} \\
& +\frac{\gamma d\left(x, x^{*}\right) d\left(x^{*}, x\right)}{1+d\left(x, x^{*}\right)+d\left(y, y^{*}\right)}
\end{aligned}
$$

so that

$$
\begin{aligned}
\left|d\left(x, x^{*}\right)\right| \leq & \left|\frac{\alpha\left(d\left(x, x^{*}\right)+d\left(y, y^{*}\right)\right)}{2}\right| \\
& +\frac{\gamma\left|d\left(x, x^{*}\right)\right|\left|d\left(x^{*}, x\right)\right|}{\left|1+d\left(x, x^{*}\right)+d\left(y, y^{*}\right)\right|} .
\end{aligned}
$$

Since $\left|1+d\left(x, x^{*}\right)+d\left(y, y^{*}\right)\right|>\left|d\left(x, x^{*}\right)\right|$, so we get

$$
\begin{aligned}
\left|d\left(x, x^{*}\right)\right| & \leq\left|\frac{\alpha\left(d\left(x, x^{*}\right)+d\left(y, y^{*}\right)\right)}{2}\right|+\gamma\left|d\left(x, x^{*}\right)\right| \\
& =\left(\frac{\alpha}{2-\alpha-2 \gamma}\right)\left|d\left(y, y^{*}\right)\right| .
\end{aligned}
$$

Similarly, one can easily prove that

$$
\left|d\left(y, y^{*}\right)\right| \leq\left(\frac{\alpha}{2-\alpha-2 \gamma}\right)\left|d\left(x, x^{*}\right)\right| .
$$

If we add (26) and (27), we get

$$
\begin{aligned}
& \left|d\left(x, x^{*}\right)\right|+\left|d\left(y, y^{*}\right)\right| \\
& \quad \leq\left(\frac{\alpha}{2-\alpha-2 \gamma}\right)\left(\left|d\left(x, x^{*}\right)\right|+\left|d\left(y, y^{*}\right)\right|\right),
\end{aligned}
$$

which is a contradiction because $\alpha+\beta+\gamma<1$. Thus, we get $x^{*}=x$ and $y^{*}=y$, which proves the uniqueness of common coupled fixed point of $S$ and $T$.

By setting $S=T$ in Theorem 10 , one deduces the following.

Corollary 11. Let $(X, d)$ be a complete complex-valued metric space, and let the mapping $T: X \times X \rightarrow X$ satisfy

$$
\begin{aligned}
& d(T(x, y), T(u, v)) \\
& \preceq \frac{\alpha(d(x, u)+d(y, v))}{2} \\
&+(\beta d(x, T(x, y)) d(u, T(u, v)) \\
&+\gamma d(u, T(x, y)) d(x, T(u, v))) \\
& \times(1+d(x, u)+d(y, v))^{-1}
\end{aligned}
$$

for all $x, y, u, v \in X$, where $\alpha, \beta$, and $\gamma$ are nonnegative reals with $\alpha+\beta+\gamma<1$. Then $T$ has a unique coupled fixed point.
Corollary 12. Let $(X, d)$ be a complete complex-valued metric space, and let the mapping $T: X \times X \rightarrow X$ satisfy

$$
\begin{aligned}
& d\left(T^{n}(x, y), T^{n}(u, v)\right) \\
& \preceq \frac{\alpha(d(x, u)+d(y, v))}{2} \\
& \quad+\left(\beta d\left(x, T^{n}(x, y)\right) d\left(u, T^{n}(u, v)\right)\right. \\
& \left.\quad+\gamma d\left(u, T^{n}(x, y)\right) d\left(x, T^{n}(u, v)\right)\right) \\
& \quad \times(1+d(x, u)+d(y, v))^{-1}
\end{aligned}
$$

for all $x, y, u, v \in X$, where $\alpha, \beta$, and $\gamma$ are nonnegative reals with $\alpha+\beta+\gamma<1$. Then, $T$ has a unique coupled fixed point.

Theorem 13. Let $(X, d)$ be a complete complex-valued metric space, and let the mappings $S, T: X \times X \rightarrow X$ satisfy

$$
\begin{aligned}
& d(S(x, y), T(u, v)) \\
& \preceq\left\{\begin{array}{lr}
\frac{\alpha(d(x, u)+d(y, v))}{2} \\
+\frac{\beta d(x, S(x, y)) d(u, T(u, v))}{d(x, T(u, v))+d(u, S(x, y))+d(x, u)+d(y, v)}, \\
0, & \text { if } D \neq 0
\end{array}\right. \\
&
\end{aligned}
$$

for all $x, y, u, v \in X$, where $D=d(x, T(u, y))+d(u, S(x, y))+$ $d(x, u)+d(y, v)$ and $\alpha, \beta$ are nonnegative reals with $\alpha+\beta<1$. Then $S$ and $T$ have a unique common coupled fixed point.

Proof. Let $x_{0}$ and $y_{0}$ be arbitrary points in $X$. Define $x_{2 k+1}=$ $S\left(x_{2 k}, y_{2 k}\right), y_{2 k+1}=S\left(y_{2 k}, x_{2 k}\right)$ and $x_{2 k+2}=T\left(x_{2 k+1}, y_{2 k+1}\right)$, $y_{2 k+2}=T\left(y_{2 k+1}, x_{2 k+1}\right)$, for $k=0,1, \ldots$

Now, we assume that

$$
\begin{aligned}
D_{S}\left(x_{2 k}, y_{2 k}\right)= & d\left(x_{2 k}, T\left(x_{2 k+1}, y_{2 k+1}\right)\right) \\
& +d\left(x_{2 k+1}, S\left(x_{2 k}, y_{2 k}\right)\right) \\
& +d\left(x_{2 k}, x_{2 k+1}\right)+d\left(y_{2 k}, y_{2 k+1}\right) \\
= & d\left(x_{2 k}, x_{2 k+2}\right)+d\left(x_{2 k}, x_{2 k+1}\right) \\
& +d\left(y_{2 k}, y_{2 k+1}\right) \neq 0, \\
D_{S}\left(y_{2 k}, x_{2 k}\right)= & d\left(y_{2 k}, T\left(y_{2 k+1}, x_{2 k+1}\right)\right) \\
& +d\left(y_{2 k+1}, S\left(y_{2 k}, x_{2 k}\right)\right) \\
& +d\left(x_{2 k}, x_{2 k+1}\right)+d\left(y_{2 k}, y_{2 k+1}\right) \\
= & d\left(y_{2 k}, y_{2 k+2}\right)+d\left(x_{2 k}, x_{2 k+1}\right) \\
& +d\left(y_{2 k}, y_{2 k+1}\right) \neq 0 .
\end{aligned}
$$


Then,

$$
\begin{aligned}
& d\left(x_{2 k+1}, x_{2 k+2}\right) \\
&= d\left(S\left(x_{2 k}, y_{2 k}\right), T\left(x_{2 k+1}, y_{2 k+1}\right)\right) \\
& \leq \frac{\alpha\left(d\left(x_{2 k}, x_{2 k+1}\right)+d\left(y_{2 k}, y_{2 k+1}\right)\right)}{2} \\
&+\frac{\beta d\left(x_{2 k}, S\left(x_{2 k}, y_{2 k}\right)\right) d\left(x_{2 k+1}, T\left(x_{2 k+1}, y_{2 k+1}\right)\right)}{D_{S}\left(x_{2 k}, y_{2 k}\right)} \\
&= \frac{\alpha\left(d\left(x_{2 k}, x_{2 k+1}\right)+d\left(y_{2 k}, y_{2 k+1}\right)\right)}{2} \\
&+\left(\beta d\left(x_{2 k}, x_{2 k+1}\right) d\left(x_{2 k+1}, x_{2 k+2}\right)\right) \\
& \quad \quad\left(d\left(x_{2 k}, x_{2 k+2}\right)+d\left(x_{2 k}, x_{2 k+1}\right)\right. \\
&\left.\quad+d\left(y_{2 k}, y_{2 k+1}\right)\right)^{-1}
\end{aligned}
$$

which implies that

$$
\begin{aligned}
& \left|d\left(x_{2 k+1}, x_{2 k+2}\right)\right| \\
& \leq \frac{\alpha\left|d\left(x_{2 k}, x_{2 k+1}\right)+d\left(y_{2 k}, y_{2 k+1}\right)\right|}{2} \\
& \quad+\left(\beta\left|d\left(x_{2 k}, x_{2 k+1}\right)\right|\left|d\left(x_{2 k+1}, x_{2 k+2}\right)\right|\right) \\
& \quad \times\left(\mid d\left(x_{2 k}, x_{2 k+2}\right)+d\left(x_{2 k}, x_{2 k+1}\right)\right. \\
& \left.\quad+d\left(y_{2 k}, y_{2 k+1}\right) \mid\right)^{-1} \\
& \leq \\
& \quad+\beta\left|d\left(x_{2 k}, x_{2 k+1}\right)+d\left(y_{2 k}, y_{2 k+1}\right)\right| \\
& 2
\end{aligned}
$$

as

$$
\begin{aligned}
& \left|d\left(x_{2 k+1}, x_{2 k+2}\right)\right| \\
& \quad \leq\left|d\left(x_{2 k+1}, x_{2 k}\right)+d\left(x_{2 k}, x_{2 k+2}\right)+d\left(y_{2 k}, y_{2 k+1}\right)\right|
\end{aligned}
$$

Therefore,

$$
\begin{aligned}
& \left|d\left(x_{2 k+1}, x_{2 k+2}\right)\right| \\
& \quad \leq \frac{(\alpha+2 \beta)}{2}\left|d\left(x_{2 k}, x_{2 k+1}\right)\right|+\frac{\alpha}{2}\left|d\left(y_{2 k}, y_{2 k+1}\right)\right|
\end{aligned}
$$

Similarly, one can easily prove that

$$
\begin{aligned}
& \left|d\left(y_{2 k+1}, y_{2 k+2}\right)\right| \\
& \quad \leq \frac{(\alpha+2 \beta)}{2}\left|d\left(y_{2 k}, y_{2 k+1}\right)\right|+\frac{\alpha}{2}\left|d\left(x_{2 k}, x_{2 k+1}\right)\right|
\end{aligned}
$$

Now, if

$$
\begin{aligned}
D_{T}( & \left.x_{2 k+1}, y_{2 k+1}\right) \\
= & d\left(x_{2 k+2}, T\left(x_{2 k+1}, y_{2 k+1}\right)\right) \\
& +d\left(x_{2 k+1}, S\left(x_{2 k+2}, y_{2 k+2}\right)\right) \\
& +d\left(x_{2 k+2}, x_{2 k+1}\right)+d\left(y_{2 k+2}, y_{2 k+1}\right) \\
= & d\left(x_{2 k+1}, x_{2 k+3}\right)+d\left(x_{2 k+2}, x_{2 k+1}\right) \\
& +d\left(y_{2 k+2}, y_{2 k+1}\right) \neq 0,
\end{aligned}
$$

we get

$$
\begin{aligned}
d\left(x_{2 k+2}, x_{2 k+3}\right) & \\
= & d\left(T\left(x_{2 k+1}, y_{2 k+1}\right), S\left(x_{2 k+2}, y_{2 k+2}\right)\right) \\
\leq & \frac{\alpha\left(d\left(x_{2 k+2}, x_{2 k+1}\right)+d\left(y_{2 k+2}, y_{2 k+1}\right)\right)}{2} \\
+ & \left(\beta d\left(x_{2 k+2}, S\left(x_{2 k+2}, y_{2 k+2}\right)\right)\right. \\
& \left.\times d\left(x_{2 k+1}, T\left(x_{2 k+1}, y_{2 k+1}\right)\right)\right) \\
& \times\left(D_{T}\left(x_{2 k+1}, y_{2 k+1}\right)\right)^{-1} \\
= & \alpha\left(d\left(x_{2 k+2}, x_{2 k+1}\right)+d\left(y_{2 k+2}, y_{2 k+1}\right)\right) \\
+ & \left(\beta d\left(x_{2 k+2}, x_{2 k+3}\right) d\left(x_{2 k+1}, x_{2 k+2}\right)\right) \\
& \times\left(d\left(x_{2 k+1}, x_{2 k+3}\right)+d\left(x_{2 k+2}, x_{2 k+1}\right)\right. \\
& \left.+d\left(y_{2 k+2}, y_{2 k+1}\right)\right)^{-1},
\end{aligned}
$$

which implies that

$$
\begin{aligned}
\left|d\left(x_{2 k+2}, x_{2 k+3}\right)\right| \\
\leq \alpha\left|\frac{\alpha\left(d\left(x_{2 k+2}, x_{2 k+1}\right)+d\left(y_{2 k+2}, y_{2 k+1}\right)\right)}{2}\right| \\
+\left(\beta\left|d\left(x_{2 k+2}, x_{2 k+3}\right)\right|\left|d\left(x_{2 k+1}, x_{2 k+2}\right)\right|\right) \\
\quad \times\left(\mid d\left(x_{2 k+1}, x_{2 k+3}\right)+d\left(x_{2 k+2}, x_{2 k+1}\right)\right. \\
\left.\quad+d\left(y_{2 k+2}, y_{2 k+1}\right) \mid\right)^{-1} \\
\leq \alpha\left|\frac{\alpha\left(d\left(x_{2 k+2}, x_{2 k+1}\right)+d\left(y_{2 k+2}, y_{2 k+1}\right)\right)}{2}\right| \\
+\beta\left|d\left(x_{2 k+1}, x_{2 k+2}\right)\right|
\end{aligned}
$$

as

$$
\begin{aligned}
& \left|d\left(x_{2 k+2}, x_{2 k+3}\right)\right| \\
& \leq \mid d\left(x_{2 k+2}, x_{2 k+1}\right)+d\left(x_{2 k+1}, x_{2 k+3}\right) \\
& \quad+d\left(y_{2 k+2}, y_{2 k+1}\right) \mid .
\end{aligned}
$$


Therefore,

$$
\begin{aligned}
\left|d\left(x_{2 k+2}, x_{2 k+3}\right)\right| & \frac{\alpha\left|d\left(x_{2 k+2}, x_{2 k+1}\right)\right|}{2}+\frac{\alpha\left|d\left(y_{2 k+2}, y_{2 k+1}\right)\right|}{2} \\
& +\beta\left|d\left(x_{2 k+1}, x_{2 k+2}\right)\right| \\
= & \frac{(\alpha+2 \beta)}{2}\left|d\left(x_{2 k+1}, x_{2 k+2}\right)\right| \\
& +\frac{\alpha}{2}\left|d\left(y_{2 k+1}, y_{2 k+2}\right)\right| .
\end{aligned}
$$

Similarly, if $D_{T}\left(y_{2 k+1}, x_{2 k+1}\right) \neq 0$, one can easily prove that

$$
\begin{aligned}
\left|d\left(y_{2 k+2}, y_{2 k+3}\right)\right| \leq & \frac{(\alpha+2 \beta)}{2}\left|d\left(y_{2 k+1}, y_{2 k+2}\right)\right| \\
& +\frac{\alpha}{2}\left|d\left(x_{2 k+1}, x_{2 k+2}\right)\right| .
\end{aligned}
$$

Adding the inequalities (36)-(43), we get

$$
\begin{aligned}
& \left|d\left(x_{2 k+1}, x_{2 k+2}\right)\right|+\left|d\left(y_{2 k+1}, y_{2 k+2}\right)\right| \\
& \quad \leq(\alpha+\beta)\left(\left|d\left(x_{2 k}, x_{2 k+1}\right)\right|+\left|d\left(y_{2 k}, y_{2 k+1}\right)\right|\right) . \\
& \left|d\left(x_{2 k+2}, x_{2 k+3}\right)\right|+\left|d\left(y_{2 k+2}, y_{2 k+3}\right)\right| \\
& \quad \leq(\alpha+\beta)\left(\left|d\left(x_{2 k+1}, x_{2 k+2}\right)\right|+\left|d\left(y_{2 k+1}, y_{2 k+2}\right)\right|\right) .
\end{aligned}
$$

If $h=(\alpha+\beta)<1$, then, from (44), we get

$$
\begin{gathered}
\left|d\left(x_{n}, x_{n+1}\right)\right|+\left|d\left(y_{n}, y_{n+1}\right)\right| \\
\leq h\left(\left|d\left(x_{n-1}, x_{n}\right)\right|+\left|d\left(y_{n-1}, y_{n}\right)\right|\right) \\
\leq \cdots \leq h^{n}\left(\left|d\left(x_{0}, x_{1}\right)\right|\right. \\
\left.+\left|d\left(y_{0}, y_{1}\right)\right|\right) .
\end{gathered}
$$

Now if $\left|d\left(x_{n}, x_{n+1}\right)\right|+\left|d\left(y_{n}, y_{n+1}\right)\right|=\delta_{n}$, then

$$
\delta_{n} \leq h \delta_{n-1} \leq \cdots \leq h^{n} \delta_{0} .
$$

Without loss of generality, we take $m>n$. Since $0 \leq h<1$, so we get

$$
\begin{aligned}
& \left|d\left(x_{n}, x_{m}\right)\right|+\left|d\left(y_{n}, y_{m}\right)\right| \\
& \leq\left|d\left(x_{n}, x_{n+1}\right)\right|+\left|d\left(y_{n}, y_{n+1}\right)\right|+\cdots \\
& \quad+\left|d\left(x_{m-1}, x_{m}\right)\right|+\left|d\left(y_{m-1}, y_{m}\right)\right| \\
& \leq\left[h^{n} \delta_{0}+h^{n+1} \delta_{0}+\cdots+h^{m-1} \delta_{0}\right] \\
& \leq \sum_{i=n}^{m-1} h^{i} \delta_{0} \longrightarrow 0, \quad \text { as } m, n \longrightarrow+\infty .
\end{aligned}
$$

This implies that $\left\{x_{n}\right\}$ and $\left\{y_{n}\right\}$ are Cauchy sequences in $X$. Since $X$ is complete, so there exists $x, y \in X$ such that $x_{n} \rightarrow x$ and $y_{n} \rightarrow y$ as $n \rightarrow+\infty$. We now show that
$x=S(x, y)$ and $y=S(y, x)$. We suppose on the contrary that $x \neq S(x, y)$ and $y \neq S(y, x)$ so that $0 \prec d(x, S(x, y))=l_{1}$ and $0 \prec d(y, S(y, x))=l_{2}$; we would then have

$$
\begin{aligned}
l_{1}= & d(x, S(x, y)) \preceq d\left(x, x_{2 k+2}\right)+d\left(x_{2 k+2}, S(x, y)\right) \\
\preceq & d\left(x, x_{2 k+2}\right)+d\left(T\left(x_{2 k+1}, y_{2 k+1}\right), S(x, y)\right) \\
\preceq & d\left(x, x_{2 k+2}\right)+\frac{\alpha\left(d\left(x_{2 k+1}, x\right)+d\left(y_{2 k+1}, y\right)\right)}{2} \\
& +\left(\beta d\left(x_{2 k+1}, T\left(x_{2 k+1}, y_{2 k+1}\right)\right) d(x, S(x, y))\right) \\
& \times\left(d\left(x_{2 k+1}, S(x, y)\right)+d\left(x, T\left(x_{2 k+1}, y_{2 k+1}\right)\right)\right. \\
& \left.\quad+d\left(x_{2 k+1}, x\right)+d\left(y_{2 k+1}, y\right)\right)^{-1} \\
\leq & d\left(x, x_{2 k+2}\right)+\frac{\alpha\left(d\left(x_{2 k+1}, x\right)+d\left(y_{2 k+1}, y\right)\right)}{2} \\
& +\left(\beta l_{1} d\left(x_{2 k+1}, x_{2 k+2}\right)\right) \\
& \times\left(d\left(x_{2 k+1}, S(x, y)\right)+d\left(x, x_{2 k+2}\right)\right. \\
& \left.\quad+d\left(x_{2 k+1}, x\right)+d\left(y_{2 k+1}, y\right)\right)^{-1}
\end{aligned}
$$

so that

$$
\begin{aligned}
\left|l_{1}\right| \leq & \left|d\left(x, x_{2 k+2}\right)\right|+\frac{\alpha}{2}\left|d\left(x_{2 k+1}, x\right)+d\left(y_{2 k+1}, y\right)\right| \\
+ & \left(\beta\left|l_{1}\right|\left|d\left(x_{2 k+1}, x_{2 k+2}\right)\right|\right) \\
\times & \left(\mid d\left(x_{2 k+1}, S(x, y)\right)+d\left(x, x_{2 k+2}\right)\right. \\
& \left.+d\left(x_{2 k+1}, x\right)+d\left(y_{2 k+1}, y\right) \mid\right)^{-1} .
\end{aligned}
$$

By taking $k \rightarrow+\infty$, we get $|d(x, S(x, y))|=0$ which is a contradiction so that $x=S(x, y)$. Now

$$
\begin{aligned}
l_{2}= & d(y, S(y, x)) \preceq d\left(y, y_{2 k+2}\right)+d\left(y_{2 k+2}, S(y, x)\right) \\
\preceq & d\left(y, y_{2 k+2}\right)+d\left(T\left(y_{2 k+1}, x_{2 k+1}\right), S(y, x)\right) \\
\preceq & d\left(y, y_{2 k+2}\right)+\frac{\alpha\left(d\left(y_{2 k+1}, y\right)+d\left(x_{2 k+1}, x\right)\right)}{2} \\
& +\left(\beta d\left(y_{2 k+1}, T\left(y_{2 k+1}, x_{2 k+1}\right)\right) d(y, S(y, x))\right) \\
& \times\left(d\left(y_{2 k+1}, S(y, x)\right)+d\left(y, T\left(y_{2 k+1}, x_{2 k+1}\right)\right)\right. \\
& \left.\quad+d\left(y_{2 k+1}, y\right)+d\left(x_{2 k+1}, x\right)\right)^{-1} \\
\preceq & d\left(y, y_{2 k+2}\right)+\frac{\alpha\left(d\left(y_{2 k+1}, y\right)+d\left(x_{2 k+1}, x\right)\right)}{2} \\
& +\left(\beta l_{2} d\left(y_{2 k+1}, y_{2 k+2}\right)\right) \\
& \times\left(d\left(y_{2 k+1}, S(y, x)\right)+d\left(y, y_{2 k+2}\right)\right. \\
& \left.\quad+d\left(y_{2 k+1}, y\right)+d\left(x_{2 k+1}, x\right)\right)^{-1},
\end{aligned}
$$


which implies that

$$
\begin{aligned}
\left|l_{2}\right| \leq & \left|d\left(y, y_{2 k+2}\right)\right|+\frac{\alpha}{2}\left|d\left(y_{2 k+1}, y\right) d\left(x_{2 k+1}, x\right)\right| \\
+ & \left(\beta\left|l_{2}\right|\left|d\left(y_{2 k+1}, y_{2 k+2}\right)\right|\right) \\
& \times\left(\mid d\left(y_{2 k+1}, S(y, x)\right)+d\left(y, y_{2 k+2}\right)\right. \\
& \left.+d\left(y_{2 k+1}, y\right)+d\left(x_{2 k+1}, x\right) \mid\right)^{-1}
\end{aligned}
$$

Which, on making $k \rightarrow+\infty$, gives us $|d(y, S(y, x))|=$ 0 which is a contradiction so that $y=S(y, x)$. It follows similarly that $x=T(x, y)$ and $y=T(y, x)$. So we have proved that $(x, y)$ is a common coupled fixed point of $S$ and $T$. As in Theorem 10, the uniqueness of common coupled fixed point remains a consequence of contraction condition (31).

We have obtained the existence and uniqueness of a unique common coupled fixed point if

$$
\begin{aligned}
& D_{S}\left(x_{2 k}, y_{2 k}\right), D_{S}\left(y_{2 k}, x_{2 k}\right), \\
& \quad D_{T}\left(x_{2 k+1}, y_{2 k+1}\right), D_{T}\left(y_{2 k+1}, x_{2 k+1}\right) \neq 0
\end{aligned}
$$

for all $k \in \mathbb{N}$. Now, assume that $D_{S}\left(x_{2 k}, y_{2 k}\right)=0$ for some $k \in \mathbb{N}$. From

$$
d\left(x_{2 k}, x_{2 k+2}\right)+d\left(x_{2 k}, x_{2 k+1}\right)+d\left(y_{2 k}, y_{2 k+1}\right)=0,
$$

we obtain that $x_{2 k}=x_{2 k+1}=x_{2 k+2}$ and $y_{2 k}=y_{2 k+1}$. If $D_{S}\left(y_{2 k}, x_{2 k}\right) \neq 0$, using (8), we deduce

$$
d\left(y_{2 k+1}, y_{2 k+2}\right)=d\left(S\left(y_{2 k}, x_{2 k}\right), T\left(y_{2 k+1}, x_{2 k+1}\right)\right)=0 \text {. }
$$

That is, $y_{2 k+1}=y_{2 k+2}$ (this equality holds also if $D_{S}\left(y_{2 k}, x_{2 k}\right)$ $=0)$. The equalities

$$
x_{2 k}=x_{2 k+1}=x_{2 k+2}, \quad y_{2 k}=y_{2 k+1}=y_{2 k+2},
$$

ensure that $\left(x_{2 k+1}, y_{2 k+1}\right)$ is a unique common coupled fixed point of $S$ and $T$. The same holds if either $D_{S}\left(y_{2 k}, x_{2 k}\right)=0$, $D_{T}\left(x_{2 k+1}, y_{2 k+1}\right)=0$, or $D_{T}\left(y_{2 k+1}, x_{2 k+1}\right)=0$.

From Theorem 13, if we assume $\alpha=0$, we obtain the following corollary.

Corollary 14. Let $(X, d)$ be a complete complex-valued metric space, and let the self-mappings $S, T: X \times X \rightarrow X$ satisfy

$$
\begin{aligned}
& d(S(x, y), T(u, v)) \\
& \preceq\left\{\begin{array}{lr}
\frac{\beta d(x, S(x, y)) d(u, T(u, v))}{d(x, T(u, v))+d(u, S(x, y))+d(x, u)+d(y, v)}, \\
0, & \text { if } D \neq 0 \\
\text { if } D \neq 0
\end{array}\right.
\end{aligned}
$$

for all $x, y, u, v \in X$, where $D=d(x, T(u, y))+d(u, S(x, y))+$ $d(x, y)+d(y, v)$ and $\beta$ is a nonnegative real such that $0<\beta<$ 1. Then $S$ and T have a unique common coupled fixed point.
Corollary 15. Let $(X, d)$ be a complete complex-valued metric space, and let the mapping $T: X \times X \rightarrow X$ satisfy

$$
\begin{aligned}
& d(T(x, y), T(u, v)) \\
& \preceq\left\{\begin{array}{lr}
\frac{\alpha(d(x, u)+d(y, v))}{2} \beta d(x, T(x, y)) d(u, T(u, v)) \\
+\frac{\beta(x, u)+d(y, v)}{d(x, T(u, v))+d(u, T(x, y))+d(x, u)}, \\
0, & \text { if } D \neq 0
\end{array},\right.
\end{aligned}
$$

for all $x, y, u, v \in X$, where $D=d(x, T(u, y))+d(u, T(x, y))+$ $d(x, u)+d(y, v)$ and $\alpha, \beta$ are nonnegative reals with $\alpha+\beta<1$. Then $T$ has a unique coupled fixed point.

Corollary 16. Let $(X, d)$ be a complete complex-valued metric space, and let the mapping $T: X \times X \rightarrow X$ satisfy

$$
\begin{aligned}
& d\left(T^{n}(x, y), T^{n}(u, v)\right) \\
& \preceq\left\{\begin{array}{lr}
\frac{\alpha(d(x, u)+d(y, v))}{2} \\
+\frac{\beta d\left(x, T^{n}(x, y)\right) d\left(u, T^{n}(u, v)\right)}{d\left(x, T^{n}(u, v)\right)+d\left(u, T^{n}(x, y)\right)+d(x, u)+d(y, v)}, \\
0, & \text { if } D \neq 0
\end{array}\right.
\end{aligned}
$$

for all $x, y, u, v \in X$, where $D=d\left(x, T^{n}(u, y)\right)+$ $d\left(u, T^{n}(x, y)\right)+d(x, u)+d(y, v)$ and $\alpha, \beta$ are nonnegative reals with $\alpha+\beta<1$. Then $T$ has a unique coupled fixed point.

Now, we furnished a nontrivial example to support our main result (Theorem 10).

Example 17. Let

$$
\begin{aligned}
& X_{1}=\{z \in \mathbb{C}: \operatorname{Re}(z) \geq 0, \operatorname{Im}(z)=0\}, \\
& X_{2}=\{z \in \mathbb{C}: \operatorname{Im}(z) \geq 0, \operatorname{Re}(z)=0\},
\end{aligned}
$$

and let $X=X_{1} \cup X_{2}$. Consider a complex valued metric $d$ : $X \times X \rightarrow \mathbb{C}$ as follows:

$$
d\left(z_{1}, z_{2}\right)=\left\{\begin{array}{r}
\frac{2}{3}\left|x_{1}-x_{2}\right|+\frac{i}{2}\left|x_{1}-x_{2}\right|, \\
\text { if } z_{1}, z_{2} \in X_{1} \\
\frac{1}{2}\left|y_{1}-y_{2}\right|+\frac{i}{3}\left|y_{1}-y_{2}\right|, \\
\quad \text { if } z_{1}, z_{2} \in X_{2} \\
\frac{i}{9}\left(x_{1}+y_{2}\right)+\frac{i}{6}\left(x_{1}+y_{2}\right), \\
\quad \text { if } z_{1} \in X_{1}, z_{2} \in X_{2} \\
\frac{i}{3}\left(x_{2}+y_{1}\right)+\frac{2 i}{9}\left(x_{2}+y_{1}\right), \\
\text { if } z_{1} \in X_{2}, z_{2} \in X_{1},
\end{array}\right.
$$


with $z_{1}=x_{1}+i y_{1}$ and $z_{2}=x_{2}+i y_{2}$. Then $(X, d)$ is a complex valued metric space. Define $S, T: X \times X \rightarrow X$ as follows:

$$
\begin{aligned}
& S\left(z_{1}, z_{2}\right)= \begin{cases}0+\frac{x_{1} x_{2}}{4} i & \text { if } z_{1}, z_{2} \in X_{1} \\
\frac{y_{1} y_{2}}{5}+0 i & \text { if } z_{1}, z_{2} \in X_{2} \\
0+\frac{x_{1} y_{2}}{8} i & \text { if } z_{1} \in X_{1} \text { and } z_{2} \in X_{2} \\
\frac{y_{1} x_{2}}{9}+0 i & \text { if } z_{1} \in X_{2} \text { and } z_{2} \in X_{1},\end{cases} \\
& T\left(z_{1}, z_{2}\right)= \begin{cases}0+\frac{x_{1} x_{2}}{6} i & \text { if } z_{1}, z_{2} \in X_{1} \\
\frac{y_{1} y_{2}}{7}+0 i & \text { if } z_{1}, z_{2} \in X_{2} \\
0+\frac{x_{1} y_{2}}{10} i & \text { if } z_{1} \in X_{1} \text { and } z_{2} \in X_{2} \\
\frac{y_{1} x_{2}}{11}+0 i & \text { if } z_{1} \in X_{2} \text { and } z_{2} \in X_{1} .\end{cases}
\end{aligned}
$$

By a routine calculation, one can easily verify that the maps $S$ and $T$ satisfy the contraction condition (8) with $\alpha=3 / 4$, $\beta=1 / 15$, and $\gamma=2 / 15$. Notice that the point $(0,0)$ remains fixed under $S$ and $T$ and is indeed unique common coupled fixed point.

\section{Conflict of Interests}

The authors declare that they have no competing interests.

\section{Authors' Contribution}

All authors contributed equally and significantly in writing this paper. All authors read and approved the final paper.

\section{Acknowledgments}

The authors thank the editor and the referees for their valuable comments and suggestions which improved greatly the quality of this paper. Marwan Amin Kutbi gratefully acknowledges the support from the Deanship of Scientific Research (DSR) at King Abdulaziz University (KAU) during this research. Cristina Di Bari is supported by Università degli Studi di Palermo (Local University Project R.S. ex 60\%).

\section{References}

[1] A. Azam, B. Fisher, and M. Khan, "Common fixed point theorems in complex valued metric spaces," Numerical Functional Analysis and Optimization, vol. 32, no. 3, pp. 243-253, 2011.

[2] J. Ahmad, M. Arshad, and C. Vetro, "On a theorem of Khan in a generalized metric space," International Journal of Analysis, vol. 2013, Article ID 852727, 6 pages, 2013.

[3] M. Arshad, A. Shoaib, and I. Beg, "Fixed point of a pair of contractive dominated mappings on a closed ball in an ordered complete dislocated metric space," Fixed Point Theory and Applications, vol. 2013, article 115, 2013.

[4] M. Arshad, J. Ahmad, and E. Karapinar, "Some common fixed point results in rectangular metric spaces," International Journal of Analysis, vol. 2013, Article ID 307234, 7 pages, 2013.
[5] M. Arshad and J. Ahmad, "On multivalued contractions in cone metric spaces without normality", The Scientific World Journal. In press.

[6] M. Arshad, E. Karapinar, and J. Ahmad, "Some unique fixed point theorem for rational contractions in partially ordered metric spaces," Journal of Inequalities and Applications, vol. 2013, article 248, 2013.

[7] H. Aydi, E. Karapınar, and W. Shatanawi, "Tripled fixed point results in generalized metric spaces," Journal of Applied Mathematics, vol. 2012, Article ID 314279, 10 pages, 2012.

[8] H. Aydi, E. Karapınar, and C. Vetro, "Meir-Keeler type contractions for tripled fixed points," Acta Mathematica Scientia B, vol. 32, no. 6, pp. 2119-2130, 2012.

[9] H. Aydi, B. Samet, and C. Vetro, "Coupled fixed point results in cone metric spaces for $\widetilde{\omega}$-compatible mappings," Fixed Point Theory and Applications, vol. 2011, article 27, 15 pages, 2011.

[10] A. Azam and M. Arshad, "Common fixed points of generalized contractive maps in cone metric spaces," Iranian Mathematical Society, vol. 35, no. 2, pp. 255-264, 2009.

[11] C. di Bari and P. Vetro, " $\varphi$-pairs and common fixed points in cone metric spaces," Rendiconti del Circolo Matematico di Palermo Series 2, vol. 57, no. 2, pp. 279-285, 2008.

[12] C. di Bari and P. Vetro, "Weakly $\varphi$-pairs and common fixed points in cone metric spaces," Rendiconti del Circolo Matematico di Palermo Series 2, vol. 58, no. 1, pp. 125-132, 2009.

[13] S. Bhatt, S. Chaukiyal, and R. C. Dimri, "Common fixed point of mappings satisfying rational inequality in complex valued metric space," International Journal of Pure and Applied Mathematics, vol. 73, no. 2, pp. 159-164, 2011.

[14] N. Hussain, M. A. Khamsi, and A. Latif, "Banach operator pairs and common fixed points in hyperconvex metric spaces," Nonlinear Analysis: Theory, Methods \& Applications, vol. 74, no. 17, pp. 5956-5961, 2011.

[15] M. A. Kutbi, J. Ahmad, N. Hussain, and M. Arshad, "Common fixed point results for mappings with rational expressions," Abstract and Applied Analysis. In press.

[16] E. Karapınar, "Some nonunique fixed point theorems of Ćirić type on cone metric spaces," Abstract and Applied Analysis, vol. 2010, Article ID 123094, 14 pages, 2010.

[17] E. Karapinar, "Couple fixed point theorems for nonlinear contractions in cone metric spaces," Computers \& Mathematics with Applications, vol. 59, no. 12, pp. 3656-3668, 2010.

[18] C. Mongkolkeha, W. Sintunavarat, and P. Kumam, "Fixed point theorems for contraction mappings in modular metric spaces," Fixed Point Theory and Applications, vol. 2011, article 93, 2011.

[19] F. Rouzkard and M. Imdad, "Some common fixed point theorems on complex valued metric spaces," Computers \& Mathematics with Applications, vol. 64, no. 6, pp. 1866-1874, 2012.

[20] W. Sintunavarat and P. Kumam, "Generalized common fixed point theorems in complex valued metric spaces and applications," Journal of Inequalities and Applications, vol. 2012, article 84, 2012.

[21] W. Sintunavarat, Y. J. Cho, and P. Kumam, "Urysohn integral equations approach by common fixed points in complex valued metric spaces," Advances in Difference Equations, vol. 2013, article 49, 2013.

[22] W. Sintunavarat and P. Kumam, "Weak condition for generalized multi-valued $(f, \alpha, \beta)$-weak contraction mappings," Applied Mathematics Letters, vol. 24, no. 4, pp. 460-465, 2011. 
[23] W. Sintunavarat, Y. J. Cho, and P. Kumam, "Common fixed point theorems for $c$-distance in ordered cone metric spaces," Computers \& Mathematics with Applications, vol. 62, no. 4, pp. 1969-1978, 2011.

[24] W. Sintunavarat and P. Kumam, "Common fixed point theorems for generalized $\mathrm{JH}$-operator classes and invariant approximations," Journal of Inequalities and Applications, vol. 2011, article 67, 2011.

[25] W. Sintunavarat and P. Kumam, "Common fixed points of $f$ weak contractors in cone metric spaces," Bulletin of the Iranian Mathematical Society, vol. 38, no. 2, pp. 293-303, 2012.

[26] N. Tahat, H. Aydi, E. Karapinar, and W. Shatanawi, "Common fixed points for single-valued and multi-valued maps satisfying a generalized contraction in G-metric spaces," Fixed Point Theory and Applications, vol. 2012, article 48, 2012.

[27] T. Gnana Bhaskar and V. Lakshmikantham, "Fixed point theorems in partially ordered metric spaces and applications," Nonlinear Analysis: Theory, Methods \& Applications, vol. 65, no. 7, pp. 1379-1393, 2006.

[28] B. Samet, E. Karapinar, H. Aydi, and V. Cojbasic, "Discussion on some coupled fixed point theorems," Fixed Point Theory and Applications, vol. 2013, article 50, 2013.

[29] B. Samet and C. Vetro, "Coupled fixed point theorems for multivalued nonlinear contraction mappings in partially ordered metric spaces," Nonlinear Analysis: Theory, Methods \& Applications, vol. 74, no. 12, pp. 4260-4268, 2011. 


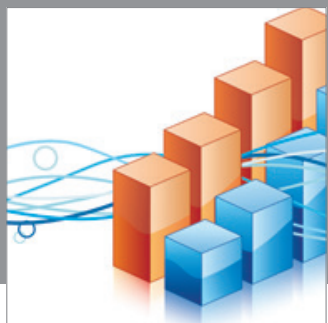

Advances in

Operations Research

mansans

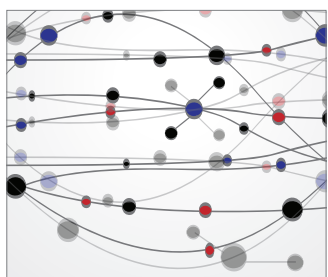

The Scientific World Journal
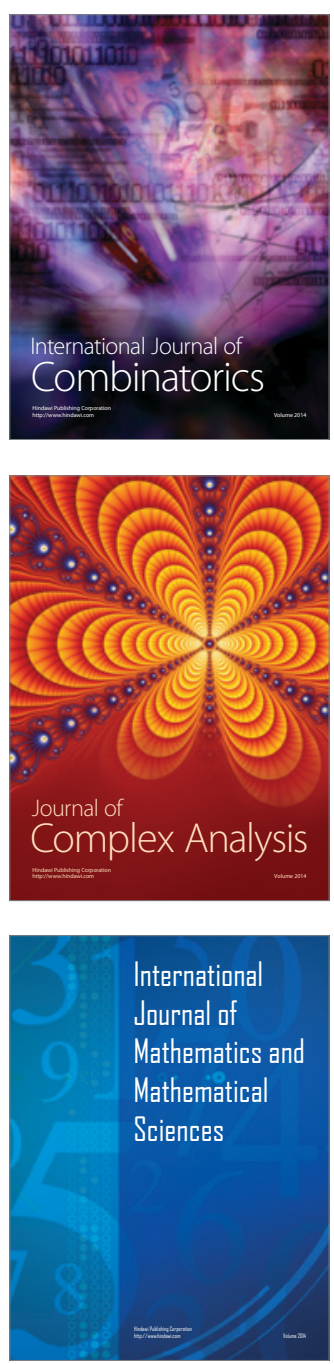
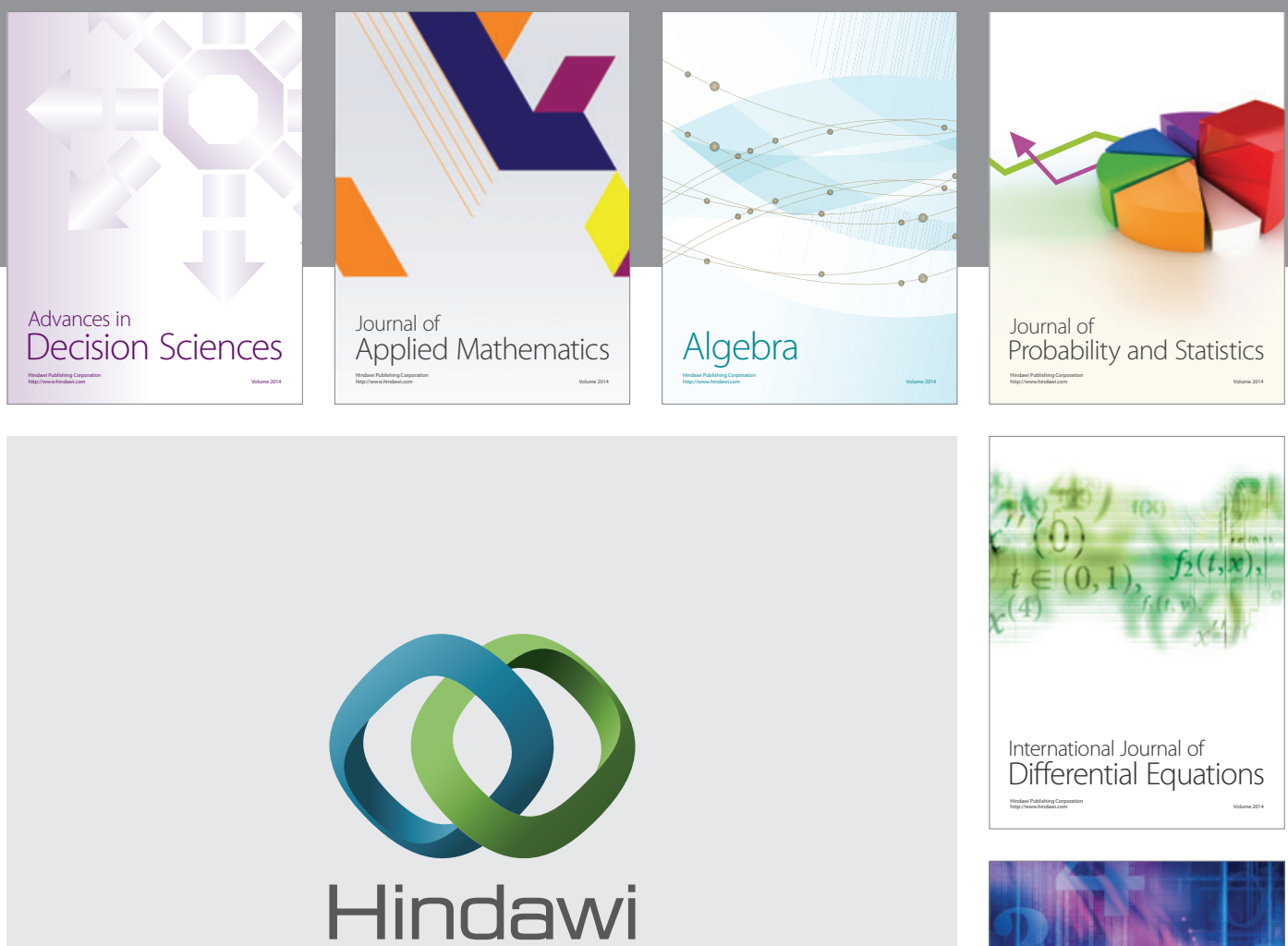

Submit your manuscripts at http://www.hindawi.com
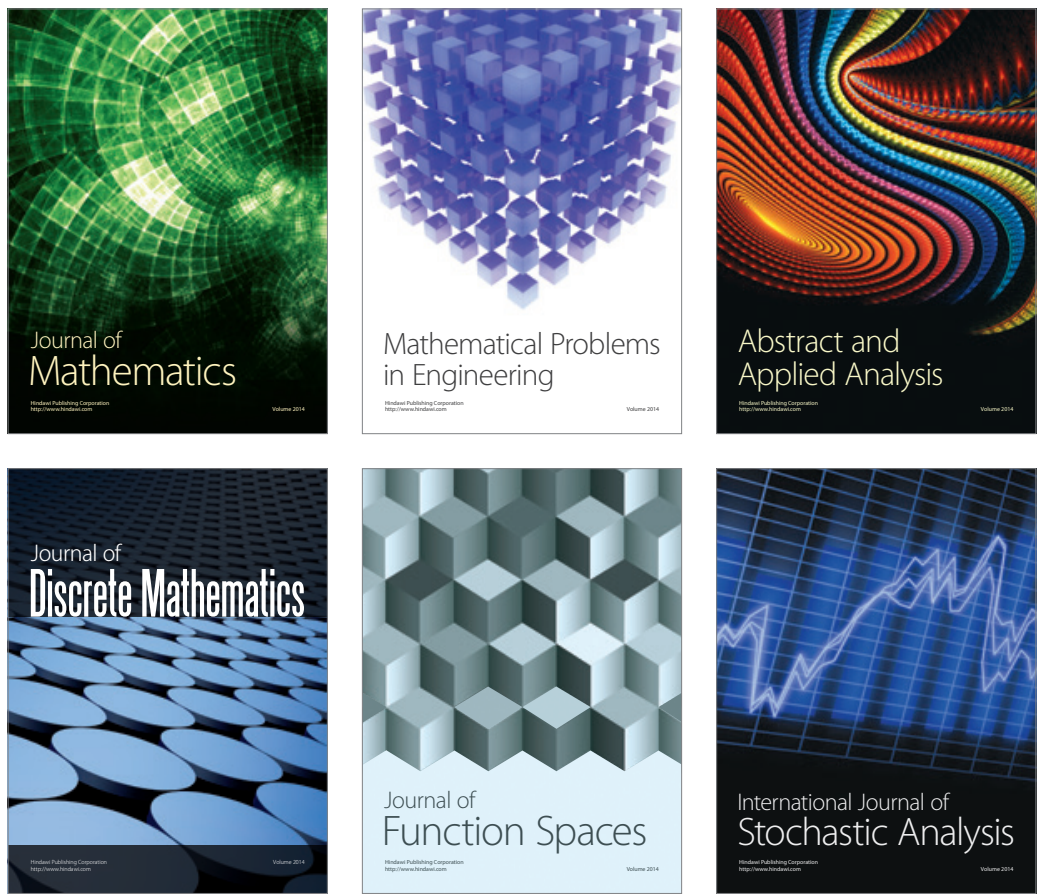

Journal of

Function Spaces

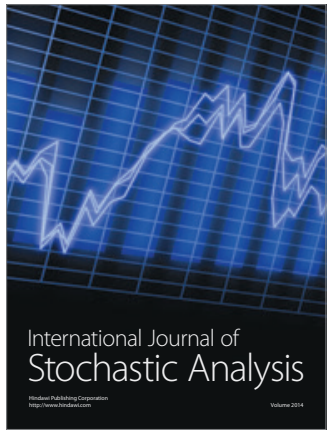

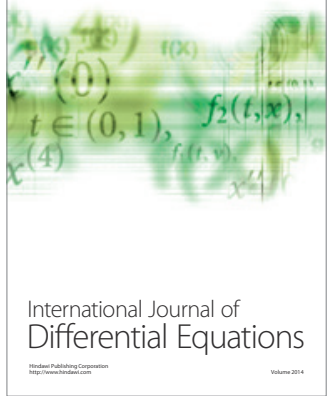
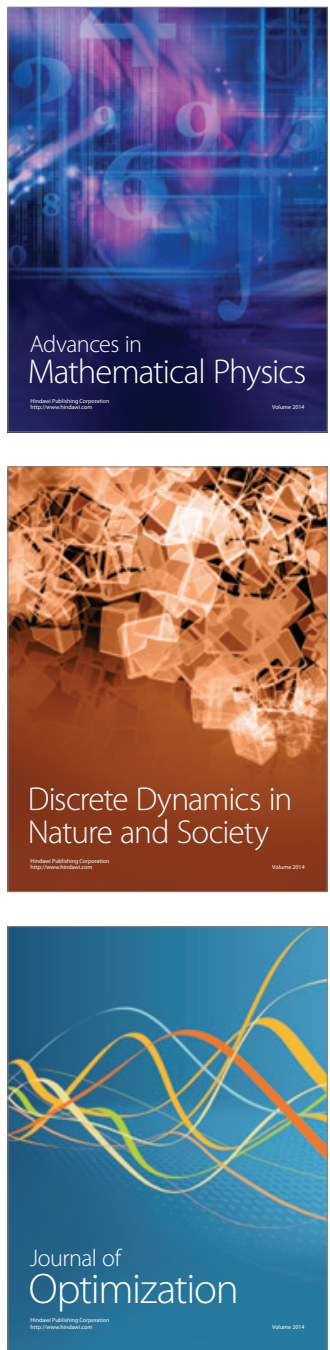